\title{
Risk assessment of diabetic foot complications in people living with T2DM using propensity score: a statistical approach
}

\author{
Ashma D Monteiro ${ }^{1}$, Shreemathi S Mayya ${ }^{1}$, Arun G Maiya ${ }^{2}$; Manipal University, Manipal, India \\ 1: Department of Statistics, Prasanna School of Public Health (PSPH) \\ 2: Department off Physiotherapy, School of Allied Health Sciences (SOAHS)
}

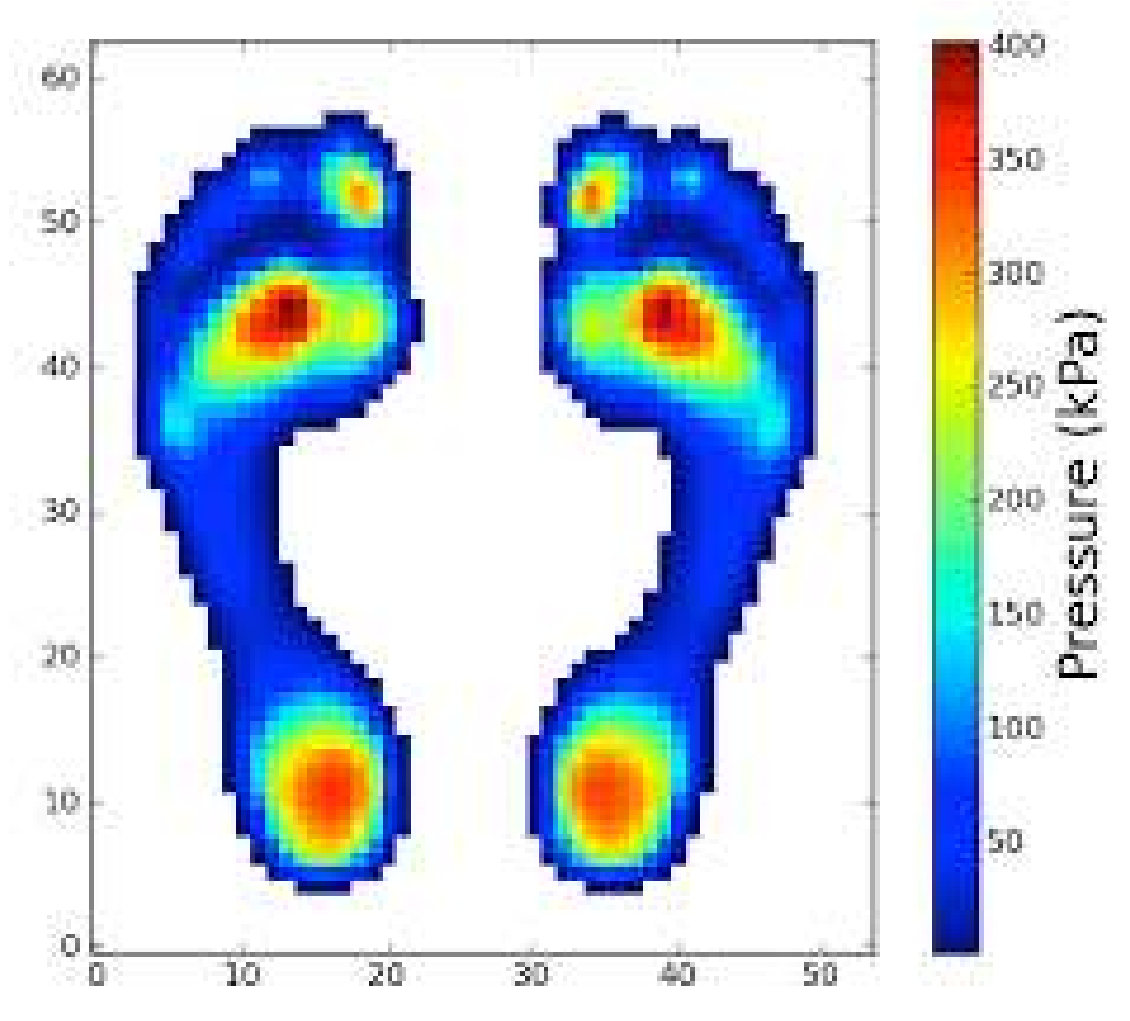

Background

Diabetic foot syndrome is characterized by several pathological conditions such as neuropathy, peripheral vascular disease, foot ulceration and infection which leads to the development of gangrene and which even necessitates limb amputations. Early detection of potential risk factors for any of the above mentioned pathological conditions can decrease the frequency of wound development. In this study, risk prediction estimates of developing diabetic neuropathy in people with T2DM is obtained, by incorporating baseline information available that may be used to improve prediction. In this study, maximum pressure which is an assessment parameter of plantar pressure, is used along with other characteristics to identify risk factors for development of peripheral neuropathy.

\section{Aims}

This study aims to assess the relationship between maximum plantar pressure and diabetic foot by adjusting cofounders using generalized propensity score (GPS) methods. Consequently we verify usage of maximum plantar pressures as risk assessment tools for diabetic foot.

\section{Methods}

A total of 214 people living with T2DM are recruited for this study, 113 of them with neuropathy and without ulceration and the rest 101 without neuropathy or ulceration (diabetic controls).

Propensity scores estimated by generalized boosting method are used to compute inverse probability weights by adjusting for other measured baseline characteristics such as age, gender, duration of diabetes, plasma glucose, body mass index and ankle-brachial index .

We then performed a weighted logistic regression analysis so as to quantify the risk of diabetic foot complications based on plantar pressure assessments in people living with T2DM..

Table 1: Absolute Correlation Coefficient (ACC) between exposure (Maximum Plantar Pressure) \& covariates

\begin{tabular}{|c|c|c|}
\hline Covariates & Original Data & GPS Weighted Data \\
\hline Age & 0.175 & 0.0093 \\
\hline Body Mass Index & 0.037 & 0.0043 \\
\hline Duration of Diabetes & 0.192 & 0.0036 \\
\hline Plasma Glucose & 0.172 & 0.0008 \\
\hline Ankle-Brachial Index & 0.080 & 0.0044 \\
\hline
\end{tabular}

\section{Results}

Imbalance observed in the measured characteristics at baseline are balanced by adjusting with inverse probability weights computed using GPS [Table 1].

$>$ The inverse probability weighted (IPW) analysis results in a more precise coefficient of exposure and hence the difference in exposure between diabetic controls and diabetic neuropathy subjects is highly significant $\left(p\right.$ value $\left.=1.21 \times 10^{-9}\right)$.

$>$ IPW analysis concludes that with ten $\mathrm{kPa}$ increase in maximum plantar pressure, the odds of developing neuropathy among people living with T2DM is expected to increase by 1.5 times with a $95 \%$ confidence interval $(1.364,1.759)$.

\section{Discussion}

Plantar pressure is a good risk assessment tool for diabetic foot syndrome. IPW analysis using GPS helps in accurately assessing risk of diabetic foot syndrome. The results of this study emphasizes on the importance to take precautionary measures to avoid complications such as peripheral neuropathy and ulceration which would further lead to amputation of foot.

\section{Bibliography}

$>$ International Diabetes Federation. IDF Diabetes Atlas. 7 ed. Brussels, Belgium: International Diabetes Federation; 2015.

$>$ Hirano K, Imbens GW. The Propensity Score with Continuous Treatments. Gelman A, Meng XL, editors. Applied Bayesian modeling and causal inference from incomplete-data perspectives. Chichester, UK: John Wiley \& Sons, Ltd, 2004; p. 73-84.

$>$ Zhu Y, Coffman DL, Ghosh D. A boosting algorithm for estimating generalized propensity scores with continuous treatments. Journal of causal inference. 2015 Mar; 3(1):25-40. 